\title{
Medication Adherence Using Electronic Monitoring in Severe Psychiatric Illness: 4 and 24 Weeks after Discharge
}

\author{
Yujin Lee, Moon-Soo Lee, Hyun-Ghang Jeong, Hyun-Chul Youn, Seung-hyun Kim \\ Department of Psychiatry, Korea University Guro Hospital, Korea University College of Medicine, Seoul, Korea
}

\begin{abstract}
Objective: The purpose of this study was to examine post-hospitalization outpatient drug adherence in patients with severe psychiatric illness, including bipolar disorder and schizophrenia, and to investigate factors associated with drug adherence.

Methods: Eighty-one patients diagnosed with schizophrenia or bipolar disorder who were hospitalized due to aggravation of psychiatric symptoms were monitored. At hospitalization, we conducted clinical assessments such as the Clinical Global Impression-Severity, Drug Attitude Inventory, Contour Drawing Rating Scale, Multidimensional Scale of Perceived Social Support scale, and patients' demographic factors. We measured drug adherence using the Medication Event Monitoring System (MEMS), pill count, and patients' self-report upon out-patients visits, 4 and 24 weeks after discharge.

Results: The mean values of the various measures of adherence were as follows: MEMS (4 weeks) $84.8 \%$, pill count (4 weeks) 94.6\%, self-report (4 weeks) 92.6\%, MEMS (24 weeks) 81.6\%, pill count (24 weeks) 90.6\%, and self-report (24 weeks) 93.6\%. The adherence agreement between MEMS, pill count, and self-report was moderate (4 weeks intraclass correlation $[\mathrm{ICC}]=0.54,24$ weeks ICC $=0.52$ ). Non-adherence (MEMS $\leq 0.08$ ) was observed in $26.4 \%$ of the patients at 4 weeks and $37.7 \%$ at 24 weeks. There was a negative correlation between drug adherence assessed 4 weeks after discharge and Contour Drawing Rating Scale difference score $(\mathrm{r}=-0.282, p<0.05)$. A positive correlation was found between drug adherence assessed 24 weeks after discharge and Drug Attitude Inventory $(\mathrm{r}=0.383, p<0.01)$. Conclusion: Patients' attitude towards their medication and their degree of physical dissatisfaction influenced post-hospitalization drug adherence in severe psychiatric patients.
\end{abstract}

KEY WORDS: Adherence; Schizophrenia; Bipolar disorder; Medication Event Monitoring System; Drug monitoring.

\section{INTRODUCTION}

Adherence can be defined as the extent to which patients follow the instructions they are given for prescribed treatments. ${ }^{1)}$ Adherence to treatment plays an essential role in achieving best outcomes in the treatment of serious mental illnesses such as schizophrenia and bipolar disorder. ${ }^{2)}$ Typical adherence rates for prescribed medication in chronic illness patients are only about $50 \%$, ${ }^{1)}$ and patients with mental illness are more likely to avoid

Received: September 7, 2018 / Revised: October 9, 2018

Accepted: October 10, 2018

Address for correspondence: Seung-hyun Kim, MD, PhD

Department of Psychiatry, Korea University Guro Hospital, Korea

University College of Medicine, 148 Gurodong-ro, Guro-gu,

Seoul 08308, Korea

Tel: +82-2-2626-1243, Fax: +82-2-852-1937

E-mail: shyun@korea.ac.kr

ORCID: https://orcid.org/0000-0002-2189-4600 taking medication than patients with other physical illnesses. ${ }^{3)}$

Schizophrenia and bipolar disorder share a number of characteristics such as their typical onset in young adults, and the frequent occurrence of live events prior to the onset, or relapse, of illness. ${ }^{4)}$ In the case of schizophrenia, it has been confirmed that the major cause of chronicity of disease progression is recurrence, if it recurs, the severity of dysfunction becomes worse and residual symptoms that did not previously exist appear. In addition, an increase in resistance to treatment results in a decrease in the effectiveness of the drug. ${ }^{5}$ In the case of bipolar disorder, drug adherence has also been shown to be consistent with reduced recurrence, and suicide rate was reported to be 5.2 times higher in patients with low adherence than that in patients with high adherence. ${ }^{6,7)}$ In patients with schizophrenia and bipolar disorder, which are

(ㄷ) This is an Open-Access article distributed under the terms of the Creative Commons Attribution Non-Commercial License (http://creativecommons.org/licenses/by-nc/4.0) which permits unrestricted non-commercial use, distribution, and reproduction in any medium, provided the original work is properly cited. 
classified as two serious mental illnesses, ${ }^{8)}$ non-adherence leads to higher rates of recurrence, re-admission, hospitalization, emergency room visits, and worsening of signs and symptoms, as well as increases in hospital costs, poorer functional outcomes and higher health care costs. $^{9-15)}$

In schizophrenic patients, the rate of non-adherence according to Medication Event Monitoring System (MEMS) results was $41.2 \%$, which was lower than the results obtained by other methods such as self-report or pill counting. ${ }^{16)}$ In bipolar disorder patients, one study reported a rate of non-adherence of up to $60 \%$ after acute episodes. ${ }^{17)}$ However, it is not easy to determine exact adherence figures in clinical practice. Adherence to drug treatment can be measured by subjective methods, such as self-report and physician report, or objective methods, such as pill counting, blood or urine analysis, electronic monitoring, and electronic refill records. ${ }^{18)}$

The subjective self-report, used mainly in the clinical setting, is often inaccurate due to memory, insight or symptoms, and the evaluation of the clinician can be inaccurate and biased because it is based on patient's selfreport. ${ }^{16)}$ Pill counts may not detect alternating under- and over-adherence or discarding of pills. ${ }^{19)}$ In order to overcome these problems, drug adherence assessment using electronic medication event monitoring is regarded as the most reliable method. ${ }^{20)}$ Technical monitors provide a quantitative estimate that is objective, independent from predictor variables and possibly less intrusive than direct observation. ${ }^{21)}$ Furthermore, the MEMS allows more precise records of drug adherence. ${ }^{22)}$

Analysis of factors related to adherence has become a critical issue for clinicians and researchers, given that identification of specific risk factors will make it possible to carry out patient-targeted interventions. ${ }^{23)}$ Factors commonly involved in non-adherence in bipolar disorder and schizophrenia patients are low level of education, young age, cognitive impairment, high intensity of delusional symptoms and suspiciousness, substance abuse/dependence, minority ethnicity, poor insight, poor therapeutic alliance, and low socioeconomic status. ${ }^{24)}$ Positive attitude to medication and illness insight were factors consistently associated with better adherence in schizophrenia patients. ${ }^{25)}$ In addition, there appears to be a significant positive association between obesity and subjective distress from weight gain and drug non-adherence. ${ }^{26)}$ In bipolar disorder, attitudes toward illness and health belief, substance and alcohol abuse, comorbid personality disorder, and side effects including weight gain, have also been suggested to be related to non-adherence. ${ }^{27-29)}$ However, there are several inconsistencies in the literature, and these relationships remain unclear. Moreover, most prior studies have limitations because their methods of assessing drug adherence have relied on subjective or indirect methods such as self-reports, provider-reports, or chart review and the study designs were retrospective or crosssectional, involving of outpatients.

We carried out 4 weeks and 24 weeks of electronic monitoring of drug adherence using MEMS. The primary aim of this prospective study was to characterize the relationship among adherence and various clinical statuses of post-discharge patients with severe psychiatric illnesses, including schizophrenia and bipolar disorder. The secondary aim was explore the characteristics of dropouts that could be classified as a wide range of non-adherence.

\section{METHODS}

\section{Participants}

Patients admitted in Korea University Guro Hospital located in Seoul between November 2012 and October 2017 were enrolled. Inclusion criteria were: a clinical diagnosis of schizophrenia or bipolar disorder according to the Diagnostic and Statistical Manual of Mental Disorders Fourth Edition (DSM-IV), and within the age range of 18 to 65 years. Exclusion criteria were comorbid diagnoses that might influence outcome measures (e.g., mental retardation), alcohol or substance dependence, neurological disease, and unstable or inadequately treated medical conditions. The study was approved by an ethical committee of the hospital and all participants provided written informed consent to participate (2012GR0028).

\section{Instruments and Assessment Procedure}

The following socio-demographic characteristics were obtained: sex, age, marital status, education level, living situation and employment status. Psychiatric history (e.g., duration of illness, age of onset, number of prior episode, duration of admission), medication history (e.g., number of medications, dose times per day), weight, height, and body mass index (BMI) were also collected.

To evaluate the severity of current psychopathology, 
Clinical Global Impression-Severity (CGI-S) scores were used. $^{30)}$ Drug attitude was measures using a Korean version of the Drug Attitude Inventory (DAI) assessment. ${ }^{31)}$ It measures patient beliefs and experience about and with medications. $^{32)}$ The Contour Drawing Rating Scale $(\mathrm{CDRS})^{33)}$ consists of a sequence of nine figures (a sequence with female silhouettes and another with male silhouettes), ordered from least to the most voluminous. Each subject selected the figure most identifiable with both their current and ideal appearance. Discrepancy between these two responses is considered an indicator of the level of dissatisfaction with body image. In addition, the Multidimensional Scale of Perceived Social Support (MSPSS) assessment, translated into Korean was used in this study. ${ }^{34)}$ MSPSS is a self-report scale of subjectively estimated social support. To evaluate cognitive function, the Korean Wechsler Intelligence Scale (K-WAIS-IV) was used.

CGI-S, DAI, CDRS, MSPSS were measured at baseline, 4 weeks and 24 weeks after discharge. Socio-demographic and clinical characteristics were assessed at baseline and body weight was assessed at each visiting. Adherence was evaluated by MEMS adherence, pill count and patient self-report at 4 weeks and 24 weeks after discharge.

\section{Medication Adherence Variables}

In this study, MEMS V TrackCap ${ }^{\circledR}$ devices (Aardex, Ltd., Zug, Switzerland) were used, and the opening and closing times were recorded in the built-in EEPROM, with the data transmitted to a computer afterwards. PowerView software (Aardex, Ltd.) was used to analyze the data. We determined the percentage of doses taken on schedule (PDTC) in this study based on the pill container being opened within a 3-hour target time frame for each dose. The PDTc was used in this study to evaluate compliance (PDTc $[\%]=$ number of doses taken correctly according to the prescription/number of prescribed doses $\times 100$ ).

Pill count adherence index was derived from the percentage of the actual pill count per subscribed pill count as recorded by the research assistants at follow-up visits. Patients were also asked to estimate their adherence to medication on a scale of $0 \%$ to $100 \%$.

\section{Statistical Analysis}

For the demographic and clinical variables, the mean value and standard deviation of the continuous variables, and the frequency and percentage of the categorical variables are presented. The chi-square test, one-way ANOVA, and Kruskal-Wallis test were used for the analysis of the drop-out group. Full scale intelligence quotient (FSIQ), baseline BMI, MSPSS Friends, and MSPSS total score were analyzed with the use of one-way ANOVA for normally distributed clinical characteristics. Age, education, duration of illness, CDRS-difference score, MSPSS Family, and MSPSS Significant other were analyzed with the use of the Kruskal-Wallis test for between-group comparisons because these variables did not achieve normality. Post-test was performed using the Mann-Whitney test. Bonferroni's method was used to determine significance levels. The degree of agreement of adherence as measured by MEMS, pill count, and the self-report was evaluated by a Bland-Altman plot. The relationship between adherence compliance and each continuous variable was verified by linear correlation with Pearson correlation analysis. All statistical analyses were performed using SPSS version 21.0 (IBM Corp., Armonk, NY, USA). Statistical significance was based on $p$ values $<0.05$.

\section{RESULTS}

\section{Demographic and Clinical Characteristics}

Out of the 81 enrolled patients, 35 (43.2\%) had been diagnosed with schizophrenia and $46(56.8 \%)$ with bipolar disorder. The mean age of patients was $36.07 \pm 12.00$ years, and $37.0 \%$ were male. The mean years of education was $13.54 \pm 2.57$, the mean IQ was 96.20 \pm 15.96 , and the mean BMI was $23.85 \pm 4.06 \mathrm{~kg} / \mathrm{m}^{2}$. In addition, $30.9 \%$ of the patients were married, and $85.2 \%$ of the patients were living with someone. The employment rate was $16.0 \%$. Patients were prescribed $8.59 \pm 3.46$ drugs (Table 1).

\section{Drop-out Analysis}

Of the first 81 patients enrolled, the 4-week follow-up adherence data were missing for 10 because 8 failed to attend visits, one did not return the MEMS bottle, and one withdrew consent. The 24-weeks follow-up adherence data were missing for 19 patients because 18 failed to attend visits between 4 and 24 weeks, and one was re-admitted. Thus, a total of 52 patients were included in the 24-week adherence analysis. The patients who drop- 
ped out in the study were considered as the non-adherent in the broad sense, and this group was included in the analysis. Patients who dropped out from the study did not differ in any characteristic from patients who completed

Table 1. Demographic and clinical characteristics of patients at baseline

\begin{tabular}{lc}
\hline \multicolumn{1}{c}{ Variable } & Patient $(\mathrm{n}=81)$ \\
\hline Sex, male & $30(37.0)$ \\
Age (yr) & $36.06 \pm 12.00$ \\
Education (yr) & $13.54 \pm 2.57$ \\
Married & $25(30.9)$ \\
Home situation (living with someone) & $69(85.2)$ \\
Currently employed & $13(16.0)$ \\
FSIQ & $96.20 \pm 15.96$ \\
BMI (kg/m ${ }^{2}$ ) & $23.85 \pm 4.06$ \\
Diagnosis, bipolar disorder & $46(56.8)$ \\
Duration of illness (mo) & $107.28 \pm 106.84$ \\
Age of onset (yr) & $28.30 \pm 10.81$ \\
Number of prior episode & $2.62 \pm 2.41$ \\
Duration of admission (day) & $34.94 \pm 15.39$ \\
Number of medications & \\
$\quad$ Total & $8.59 \pm 3.46$ \\
$\quad$ Psychiatric & $7.43 \pm 2.61$ \\
Dose times per day & \\
$\quad$ Total & $2.22 \pm 1.14$ \\
$\quad$ Psychiatric & $1.75 \pm 0.43$ \\
\hline
\end{tabular}

Values are presented as number (\%) or mean \pm standard deviation. $\mathrm{FSIQ}$, full scale intelligence quotient; BMI, body mass index. follow-up period.

However, the years of education and IQ were lower in patients who dropped out in less than 4 weeks. In the drop-out group, the baseline BMI was higher than that of the maintenance group. In addition, the mean CGI-S score was higher and the mean DAI score was lower in the drop-out group compared to the scores in the other group. The CDRS-difference score was lower in the group with less than 4 weeks of follow-up compared to the scores in the group with less than 24 weeks of follow-up and with the maintenance group (Table 2).

\section{Medication Adherence}

The mean values of the various measures of adherence were as follows: MEMS (4 weeks) $84.8 \pm 19.8 \%$, pill count (4 weeks) $94.6 \pm 12.0 \%$, self-report (4 weeks) $92.6 \pm 10.7 \%$, MEMS (24 weeks) $81.6 \pm 23.2 \%$, pill count (24 weeks) $90.6 \pm 17.2 \%$, and self-report (24 weeks) $93.6 \pm 9.7 \%$. The adherence agreement between MEMS, pill count, and self-report was moderate (4 weeks intraclass correlation $[$ ICC $]=0.54,24$ weeks ICC $=0.52$ ). Non-adherence (MEMS $\leq 0.08$ ) was observed in $26.4 \%$ of the patients at 4 weeks and $37.7 \%$ at 24 weeks.

Figure $1 \mathrm{~A}$ shows the Bland-Altman plot of MEMS and self-report adherence, with a bias of $-7.9 \%$ (95\% limit of agreement, $-48.7 \%$ to $33.0 \%)$. Figure $1 \mathrm{~B}$ shows the

Table 2. Comparison of dropped out within 4 weeks, dropped out between 4 weeks and 24 weeks, and 24 weeks follow-up patients

\begin{tabular}{|c|c|c|c|c|}
\hline Variable & $<4$ weeks $(n=10)$ & $<24$ weeks $(\mathrm{n}=19)$ & 24 weeks ( $n=52$ ) & $p$ value \\
\hline Diagnosis & & & & $0.963^{*}$ \\
\hline Schizophrenia & $3(70.0)$ & $11(57.9)$ & $21(40.4)$ & \\
\hline Bipolar disorder & $7(30.0)$ & $8(42.1)$ & 31 (59.6) & \\
\hline Age (yr) & $33.00 \pm 5.12$ & $37.83 \pm 3.30$ & $34.29 \pm 1.75$ & 0.147 \\
\hline Education (yr) & $12.75 \pm 0.75$ & $13.17 \pm 0.72$ & $14.22 \pm 0.36$ & 0.305 \\
\hline FSIQ & $90.00 \pm 13.08$ & $96.50 \pm 15.51$ & $97.09 \pm 16.60$ & 0.557 \\
\hline Duration of illness (yr) & $63.00 \pm 22.83$ & $147.50 \pm 42.59$ & $97.63 \pm 14.97$ & 0.524 \\
\hline Baseline BMI (kg/m²) & $25.15 \pm 3.29$ & $25.71 \pm 1.66$ & $22.86 \pm 0.48$ & 0.890 \\
\hline CGI-S & $3.25 \pm 0.95$ & $2.67 \pm 0.40$ & $2.83 \pm 0.16$ & 0.943 \\
\hline DAI & $0.75 \pm 1.25$ & $3.00 \pm 1.51$ & $3.42 \pm 0.66$ & 0.385 \\
\hline CDRS-difference score & $4.50 \pm 2.87$ & $4.46 \pm 1.59$ & $3.15 \pm 0.64$ & 0.403 \\
\hline \multicolumn{5}{|l|}{ MSPSS } \\
\hline Family & $23.25 \pm 2.75$ & $20.75 \pm 1.79$ & $21.44 \pm 0.91$ & 0.854 \\
\hline Friends & $19.25 \pm 4.78$ & $17.33 \pm 1.68$ & $18.07 \pm 1.02$ & 0.968 \\
\hline Significant other & $20.25 \pm 4.97$ & $17.83 \pm 2.05$ & $19.66 \pm 1.00$ & 0.785 \\
\hline Total score & $62.75 \pm 12.46$ & $55.08 \pm 5.01$ & $59.10 \pm 2.52$ & 0.805 \\
\hline
\end{tabular}

Values are presented as number (\%) or mean \pm standard deviation.

FSIQ, full scale intelligence quotient; BMI, body mass index; CGI-S, Clinical Global Impression-Severity; DAI, Drug Attitude Inventory; CDRS, Contour Drawing Rating Scale; MSPSS, Multidimensional Scale of Perceived Social Support.

$p$ values were calculated using one-way ANOVA, Kruskal-Wallis $\mathrm{H}$ test, and *chi-square test for linear by linear association. 

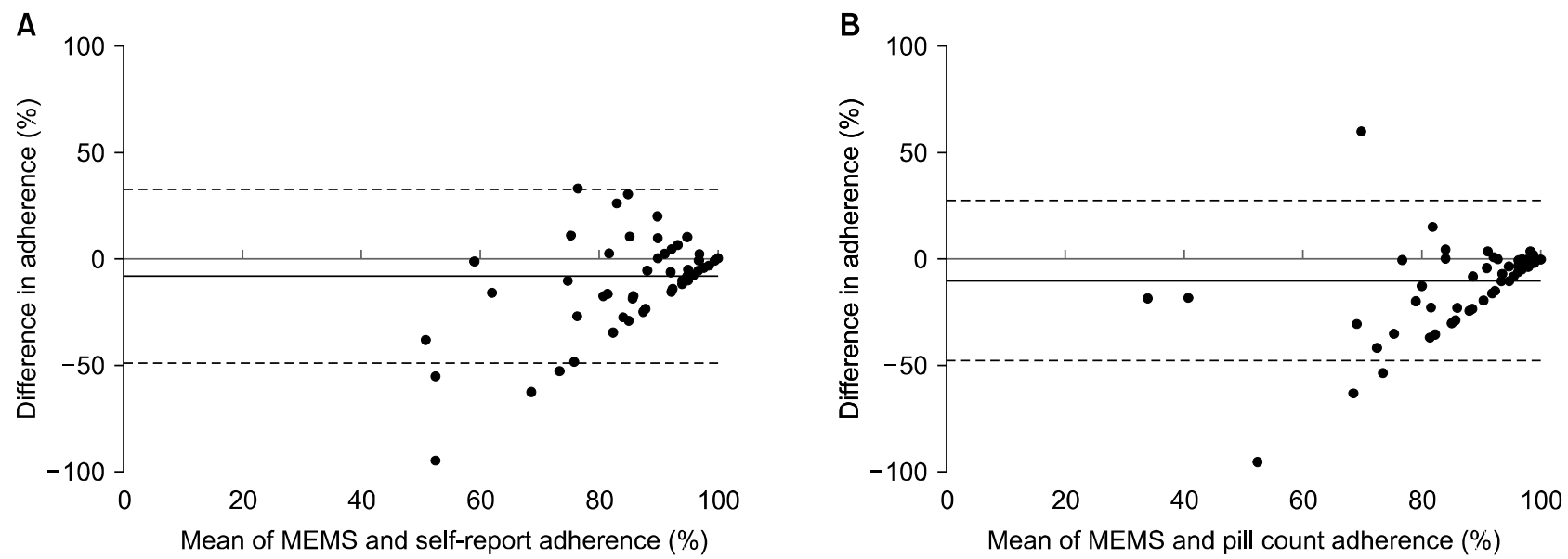

Fig. 1. Bias analysis of drug adherence measured by Medication Event Monitoring System (MEMS), pill count and self-report. (A) Bland-Altman plot of adherence as measured by 4-week MEMS adherence and self-report adherence. (B) Bland-Altman plot of adherence as measured by 4-week MEMS adherence and pill count adherence.

Dotted lines, 95\% limits of agreement.

Table 3. Correlations between Medication Electronic Monitoring System (MEMS) adherence and clinical variables

\begin{tabular}{|c|c|c|c|c|}
\hline \multirow{3}{*}{ Variable } & \multicolumn{4}{|c|}{ MEMS adherence } \\
\hline & \multicolumn{2}{|c|}{4 weeks $(n=71)$} & \multicolumn{2}{|c|}{24 weeks ( $n=52)$} \\
\hline & $r$ & $p$ value & $\mathrm{r}$ & $p$ value \\
\hline Age $(y r)$ & 0.044 & 0.711 & -0.108 & 0.442 \\
\hline Education (yr) & 0.150 & 0.208 & 0.089 & 0.525 \\
\hline FSIQ & 0.033 & 0.804 & 0.016 & 0.918 \\
\hline Duration of illness (mo) & -0.057 & 0.634 & -0.149 & 0.287 \\
\hline \multicolumn{5}{|l|}{ Number of medications } \\
\hline Total & -0.037 & 0.760 & -0.065 & 0.645 \\
\hline Psychiatric & -0.048 & 0.688 & 0.097 & 0.487 \\
\hline \multicolumn{5}{|l|}{ Dose times per day } \\
\hline Total & -0.068 & 0.568 & 0.026 & 0.853 \\
\hline Psychiatric & -0.024 & 0.841 & 0.064 & 0.647 \\
\hline Duration of admission (day) & -0.038 & 0.753 & 0.056 & 0.689 \\
\hline Number of prior episode & -0.090 & 0.453 & -0.030 & 0.831 \\
\hline Age of onset (yr) & 0.171 & 0.152 & 0.001 & 0.999 \\
\hline Baseline BMI $\left(\mathrm{kg} / \mathrm{m}^{2}\right)$ & -0.218 & 0.074 & -0.160 & 0.263 \\
\hline CGI-S & -0.062 & 0.614 & 0.167 & 0.238 \\
\hline DAI & 0.031 & 0.796 & 0.383 & $0.005^{* *}$ \\
\hline CDRS-difference score & -0.282 & $0.016^{*}$ & 0.031 & 0.823 \\
\hline \multicolumn{5}{|l|}{ MSPSS } \\
\hline Family & -0.091 & 0.448 & -0.154 & 0.274 \\
\hline Friends & -0.164 & 0.173 & -0.144 & 0.309 \\
\hline Significant other & -0.178 & 0.138 & -0.154 & 0.275 \\
\hline Total score & -0.175 & 0.144 & -0.173 & 0.219 \\
\hline
\end{tabular}

FSIQ, full scale intelligence quotient; BMI, body mass index; GCI-S, Clinical Global Impression-Severity; DAI, Drug Attitude Inventory; CDRS, Contour Drawing Rating Scale; MSPSS, Multidimensional Scale of Perceived Social Support.

$p$ values are calculating using Pearson's correlation analysis and Spearman's correlation analysis.

${ }^{*} p<0.05,{ }^{* *} p<0.01$.

Bland-Altman plot of MEMS and pill count adherence, with a bias of $-9.9 \%(95 \%$ limit of agreement, $-47.9 \%$ to $28.1 \%$ ). These results indicate a great differences between
MEMS adherence and self-report, and the pill count results. 


\section{Relationships between MEMS Adherence and Variables}

In the correlation analysis, the CDRS-difference score was negatively correlated with 4 weeks MEMS adherence $(r=-0.282, p=0.016)$ and the DAI score was positively correlated with 24 weeks MEMS adherence $(r=0.383$, $p=0.005$ ).

Adherence did not differ according to gender, age, education, duration of illness, frequency of medication, or any other demographic factor. MEMS adherence was not related to CGI-S or MSPSS score (Table 3).

\section{DISSCUSION}

The significance of the present study stems from the fact that it was a 24-week long-term prospective study on drug adherence and related factors among in-patients with severe psychiatric illness after discharge.

The average drug adherence as measured by MEMS at 4 weeks and 24 weeks after discharge in the present study was $82.4 \%$ and $81.6 \%$ respectively. With $80 \%$ adherence as the standard for drug adherence, non-adherence at 4 weeks post-discharge was $26.4 \%$, while non-adherence at 24 weeks post-discharge reached $37.7 \%$. This result is similar to that of existing studies based on MEMS. Recent studies on schizophrenia using MEMS reported an average non-adherence rate during 6 months as $62.7 \%$, and studies on bipolar disorder reported non-adherence rates of $20 \%$ to $60 \% .^{27,35-37)}$ In this study, drug adherence measured by MEMS was found to be lower than that measured by pill count or self-report. Drug adherence measured by MEMS and self-report showed a particularly large significance difference. According to existing literature, the drug adherence rate among patients with schizophrenia and schizoaffective disorders estimated by objective measures was $65.5 \%,{ }^{12)} 60 \%$ to $81 \%$ by a combination of subjective and objective measures, ${ }^{38,39)}$ and $34 \%$ to $80 \%$ by subjective measures alone, ${ }^{40-42)}$ revealing a large degree of difference among results. When using various measures within a single study, ${ }^{43)}$ the drug adherence rate estimated by self-report was $92 \%, 71 \%$ by pill- count, and $73 \%$ by MEMS. Another study ${ }^{16)}$ found the rate of non-adherence to be $25.5 \%$ estimated by self-report, $7.8 \%$ by pill-count, and $42.5 \%$ by MEMS, findings which are consistent with the outcomes of this study, and revealing the lowest drug adherence by the MEMS. Such a result may reflect an overestimation of adherence among patients aware of the fact that their behaviors are assessed with the utilization of the MEMS device, ${ }^{44)}$ or due to the over-report by patients through self-report or pill count. ${ }^{45,46)}$ Considering that MEMS is generally used as the reference standard for drug adherence, ${ }^{21,47)}$ nonetheless, these results are consistent with the existing literature revealing that self-report and pill count may misestimate patients' actual drug adherence. ${ }^{48)}$ Such findings imply that patients' self-report and pill count results may lead to the overestimation of their actual drug adherence in clinical practice. In clinical practice, procedural complications and expense prohibits the application of electronic monitoring devices such as MEMS in all patients. Clinicians therefore should consider the fact that drug compliance may be estimated differently according to each type of assessment when they use simpler methods such as self-report and pill count.

In this study, drug adherence at 4 weeks after discharge showed a significant negative correlation with the CDRSdifference score of physical dissatisfaction, similar to the existing reports. In all patients reporting different CDRS scores, the actual body image was larger, and patients with higher drug adherence reported their actual body image to be more similar to their desired slimmer physique. Although not statistically significant, analysis of the drop-out group revealed the largest CRDS-difference score in the drop-out group at 4 weeks, followed by an intermediate score in the drop-out group between 4 and 24 weeks, and the smallest score in the adherence group. However, the CDRS-difference score was not significantly correlated with 24-week drug adherence. The mean BMI in the drop-out group was higher than $25 \mathrm{~kg} / \mathrm{m}^{2}$, which was classified as obesity, but $22.86 \pm 0.48 \mathrm{~kg} / \mathrm{m}^{2}$ in the 24-week maintenance group, which was relatively low. Therefore, the withdrawal of patients with obesity and high levels of dissatisfaction with their body image may be the cause of the correlation with the 4-week drug adherence alone. The present study, unlike those in the existing literature, did not reveal a significant correlation between the baseline BMI at discharge and drug adherence thereafter, which may be influenced by the fact that the BMI of drop-out group which is sometimes categorized as nonadherence group in the broader sense, was high enough to be considered as overweight. Another factor is that in patients with serious psychiatric conditions beginning 
hospitalization, the types of medication may be changed and doses increased, which can lead to more side effects and physical dissatisfaction. There is need for further research on the influence of physical dissatisfaction on drug adherence, using both objective measures such as weight gain during hospitalization and the resulting difference in $\mathrm{BMI}$, and subjective measures such as CDRS. DAI, which measures patients' attitudes towards their medication, was the only measure showing significant positive correlation with the relatively long-term 24-week drug adherence. In a study on drug compliance among psychiatric outpatients, the total DAI tended to show lower score in noncompliance group, ${ }^{49,50)}$ similar to the result in the present study. However, there was no significant correlation between DAI and MEMS adherence at 4 weeks after discharge. This also seems to have affected the drop-out group as shown in the CDRS-difference score. As shown in Table 2, analysis revealed the lowest DAI score in the drop-out group at 4 weeks, followed by an intermediate score in the drop-out group at 4 to 24 weeks, and the highest score in the maintenance group. This suggests that patients who dropped out early may have poor attitudes toward medication. Therefore, negative attitudes toward medication can be a cause of treatment discontinuation after discharge, as well as lower MEMS drug adherence in patients participating in this study with outpatient follow-up for 24 weeks after discharge. It seems reasonable to expect, therefore, that comprehending the patients' negative perception about their medication, including such factors as weight gain or physical dissatisfaction, and working to resolve such issues, may be an effective method to improve long-term treatment adherence. Precedent studies have also suggested that psychoeducation that includes information about the patients' disorder, treatment, relapse prevention, medication, and its side effects is crucial in the modification of patients' attitude toward medication. ${ }^{32)}$ This study, however, found no significant correlation between drug adherence and age, sex, education level, duration of illness, social support, or medication schedule. This result is inconsistent with the existing studies presenting factors associated with drug non-adherence in both schizophrenia and bipolar disorder, such as young age, low education level, cognitive decline, severity of delusional symptoms, drug abuse and dependence, poor insight, poor therapeutic alliance, low socioeconomic status, and negative inpatient experi- ences. ${ }^{27)}$ Further large scale studies may be of importance, considering that there are conflicting studies about the influence of familial conflicts on treatment adherence, some showing a negative influence ${ }^{51,52)}$ and some showing a no influence. ${ }^{44)}$

The first limitation of this study is its relatively small sample, which may prevent generalization of the study results. The second limitation of this study is its high drop-out rate. This may pose a limitation in that a part of the drop-out group was the non-adherent group, which included patients who dropped out of the study. Finally, although MEMS is used as the reference standard for drug adherence, the possibility that patients may open the lid and still not take the drug may result in a discrepancy between the actual and recorded drug adherence.

Despite these limitations, this study has an advantage in that it a prospective study that examined objective outpatient drug adherence using a relatively established measurement, MEMS, among patients with severe psychiatric illness over a period of 24 weeks after discharge.

Study examined post-discharge outpatient drug adherence and factors influencing adherence among patients with severe psychiatric illness including schizophrenia and bipolar disorder. According to the results, significant correlations were found between drug adherence at 4 weeks after discharge and the CDRS-difference score, as well as between drug adherence at 24 weeks after discharge and the DAI score. Physical dissatisfaction at discharge may affect short-term drug adherence, whereas attitudes towards medication affect long-term drug adherence. Thus, it may be possible to increase post-discharge drug adherence among patients with severe psychiatric illness by considering factors that influence drug adherence after hospitalization and implementing appropriate interventions. These factors include patients' physical dissatisfaction and negative attitudes toward medications during hospitalization.

\section{- Acknowledgments}

This study was supported by Hanlim Pharmaceuticals (Prof. Kim). The manuscript includes parts of a thesis by Yujin Lee.

\section{REFERENCES}

1. Haynes RB, McDonald H, Garg AX, Montague P. Interventions for helping patients to follow prescriptions for 
medications. Cochrane Database Syst Rev 2002;(2):CD000011.

2. Velligan DI, Lam F, Ereshefsky L, Miller AL. Psychopharmacology: perspectives on medication adherence and atypical antipsychotic medications. Psychiatr Serv 2003;54:665-667.

3. Blackwell B. Patient compliance. N Engl J Med 1973;289: 249-252.

4. Murray RM, Sham P, Van Os J, Zanelli J, Cannon M, McDonald C. A developmental model for similarities and dissimilarities between schizophrenia and bipolar disorder. Schizophr Res 2004;71:405-416.

5. Lieberman JA, Murray R. Comprehensive care of schizophrenia: a textbook of clinical management. 2nd ed. New York:Oxford University Press;2012.

6. Altman S, Haeri S, Cohen LJ, Ten A, Barron E, Galynker II, et al. Predictors of relapse in bipolar disorder: a review. J Psychiatr Pract 2006; 12:269-282.

7. Gonzalez-Pinto A, Mosquera F, Alonso M, López P, Ramírez $\mathrm{F}$, Vieta E, et al. Suicidal risk in bipolar / disorder patients and adherence to long-term lithium treatment. Bipolar Disord 2006;8:618-624.

8. Jónsdóttir $H$, Opjordsmoen $S$, Birkenaes $A B$, Simonsen $C$, Engh JA, Ringen PA, et al. Predictors of medication adherence in patients with schizophrenia and bipolar disorder. Acta Psychiatr Scand 2013;127:23-33.

9. Svarstad BL, Shireman TI, Sweeney JK. Using drug claims data to assess the relationship of medication adherence with hospitalization and costs. Psychiatr Serv 2001;52:805-811.

10. Scott J, Pope M. Nonadherence with mood stabilizers: prevalence and predictors. J Clin Psychiatry 2002;63:384-390.

11. Jeste SD, Patterson TL, Palmer BW, Dolder CR, Goldman S, Jeste DV. Cognitive predictors of medication adherence among middle-aged and older outpatients with schizophrenia. Schizophr Res 2003;63:49-58.

12. Lindenmayer JP, Liu-Seifert H, Kulkarni PM, Kinon BJ, Stauffer $\mathrm{V}$, Edwards SE, et al. Medication nonadherence and treatment outcome in patients with schizophrenia or schizoaffective disorder with suboptimal prior response. J Clin Psychiatry 2009;70:990-996.

13. Higashi K, Medic G, Littlewood KJ, Diez T, Granström O, De Hert M. Medication adherence in schizophrenia: factors influencing adherence and consequences of nonadherence, a systematic literature review. Ther adv psychopharmacol 2013;3:200-218.

14. Robinson D, Woerner MG, Alvir JM, Bilder R, Goldman R, Geisler $\mathrm{S}$, et al. Predictors of relapse following response from a first episode of schizophrenia or schizoaffective disorder. Arch Gen Psychiatry 1999;56:241-247.

15. Haywood TW, Kravitz HM, Grossman LS, Cavanaugh JL Jr, Davis JM, Lewis DA. Predicting the "revolving door" phenomenon among patients with schizophrenic, schizoaffective, and affective disorders. Am J Psychiatry 1995; 152:856-861.

16. Yang J, Ko YH, Paik JW, Lee MS, Han C, Joe SH, et al. Symptom severity and attitudes toward medication: impacts on adherence in outpatients with schizophrenia. Schizophr Res 2012;134:226-231.

17. Geddes JR, Miklowitz DJ. Treatment of bipolar disorder. Lancet 2013;381:1672-1682.

18. Bellack AS, Bowden CL, Bowie CR, Byerly MJ, Carpenter WT, Copeland LA, et al. The expert consensus guideline series: adherence problems in patients with serious and persistent mental illness. J Clin Psychiatry 2009;70:1-48.

19. Cramer JA, Mattson RH, Prevey ML, Scheyer RD, Ouellette VL. How often is medication taken as prescribed? A novel assessment technique. JAMA 1989;261:3273-3277.

20. Saini SD, Schoenfeld P, Kaulback K, Dubinsky MC. Effect of medication dosing frequency on adherence in chronic diseases. Am J Manag Care 2009;15:e22-e33.

21. Diaz E, Levine HB, Sullivan MC, Sernyak MJ, Hawkins KA, Cramer JA, et al. Use of the Medication Event Monitoring System to estimate medication compliance in patients with schizophrenia. J Psychiatry Neurosci 2001;26:325-329.

22. George CF, Peveler RC, Heiliger S, Thompson C. Compliance with tricyclic antidepressants: the value of four different methods of assessment. Br J Clin Pharmacol 2000;50:166-171.

23. Remington G, Teo C, Mann S, Hahn M, Foussias G, Agid O. Examining levels of antipsychotic adherence to better understand nonadherence. J Clin Psychopharmacol 2013;33:261263.

24. García S, Martínez-Cengotitabengoa M, López-Zurbano S, Zorrilla I, López P, Vieta E, et al. Adherence to antipsychotic medication in bipolar disorder and schizophrenic patients: a systematic review. J Clin Psychopharmacol 2016;36:355371.

25. Sendt KV, Tracy DK, Bhattacharyya S. A systematic review of factors influencing adherence to antipsychotic medication in schizophrenia-spectrum disorders. Psychiatry Res 2015;225: 14-30.

26. Weiden PJ, Mackell JA, McDonnell DD. Obesity as a risk factor for antipsychotic noncompliance. Schizophr Res 2004;66: 51-57.

27. Lingam R, Scott J. Treatment non-adherence in affective disorders. Acta Psychiatr Scand 2002;105:164-172.

28. Vieta E. Improving treatment adherence in bipolar disorder through psychoeducation. I Clin Psychiatry 2005;66 Supp/ 1:24-29.

29. Colom F, Vieta E, Tacchi MJ, Sánchez-Moreno J, Scott J. Identifying and improving non-adherence in bipolar disorders. Bipolar Disord 2005; 7 Supp/ 5:24-31.

30. Guy W. Clinical global impression. In: Guy W, editor. ECDEU assessment manual for psychopharmacology: 1976. Rockville:National Institute of Mental Health;1976. p.217-222.

31. Yoon BH, Bahk WM, Lee KU, Hong CH, Ahn JK, Kim MK. Psychometric properties of Korean version of drug attitude inventory(KDAl-10). Korean J Psychopharmacol 2005;16:480487. 
32. Freudenreich O, Cather C, Evins AE, Henderson DC, Goff DC. Attitudes of schizophrenia outpatients toward psychiatric medications: relationship to clinical variables and insight. J Clin Psychiatry 2004;65:1372-1376.

33. Thompson MA, Gray JJ. Development and validation of a new body-image assessment scale. J Pers Assess 1995;64:258269.

34. Shin JS, Lee YB. The effects of social supports on psychosocial well-being of the unemployed. Korean I Soc Welf 1999;37: 241-269.

35. Colom F, Vieta E, Martínez-Arán A, Reinares M, Benabarre A, Gastó C. Clinical factors associated with treatment noncompliance in euthymic bipolar patients. J Clin Psychiatry 2000;61:549-555.

36. Sajatovic M, Valenstein M, Blow FC, Ganoczy D, Ignacio RV. Treatment adherence with antipsychotic medications in bipolar disorder. Bipolar Disord 2006;8:232-241.

37. Licht RW, Vestergaard P, Rasmussen NA, Jepsen K, Brodersen A, Hansen PE. A lithium clinic for bipolar patients: 2-year outcome of the first 148 patients. Acta Psychiatr Scand 2001; 104:387-390.

38. Martinez-Aran A, Scott J, Colom F, Torrent C, TabaresSeisdedos R, Daban C, et al. Treatment nonadherence and neurocognitive impairment in bipolar disorder. J Clin Psychiatry 2009;70:1017-1023.

39. Miller R, Ream G, McCormack J, Gunduz-Bruce H, Sevy S, Robinson D. A prospective study of cannabis use as a risk factor for non-adherence and treatment dropout in first-episode schizophrenia. Schizophr Res 2009;113:138-144.

40. Karow A, Czekalla J, Dittmann RW, Schacht A, Wagner T, Lambert M, et al. Association of subjective well-being, symptoms, and side effects with compliance after 12 months of treatment in schizophrenia. J Clin Psychiatry 2007;68:75-80.

41. Lacro JP, Dunn LB, Dolder CR, Leckband SG, Jeste DV. Prevalence of and risk factors for medication nonadherence in patients with schizophrenia: a comprehensive review of recent literature. J Clin Psychiatry 2002;63:892-909.

42. van Nimwegen-Campailla L, van Beveren N, Laan W, van den Brink W, Linszen D, de Haan L. Effect of early dysphoric response and cannabis use on discontinuation of olanzapine or risperidone in patients with early psychosis. Pharmacopsychiatry 2010:43:281.

43. Brain C, Allerby K, Sameby B, Quinlan P, Joas E, Karilampi U, et al. Drug attitude and other predictors of medication adherence in schizophrenia: 12 months of electronic monitoring (MEMS(R)) in the Swedish COAST-study. Eur Neuropsychopharmacol 2013;23:1754-1762.

44. Nakonezny PA, Byerly MJ. Electronically monitored adherence in outpatients with schizophrenia or schizoaffective disorder: a comparison of first- vs. second-generation antipsychotics. Schizophr Res 2006;82:107-114.

45. Sajatovic M, Chen P, Dines P, Shirley ER. Psychoeducational approaches to medication adherence in patients with bipolar disorder. Dis Manag Health Outcomes 2007;15:181-192.

46. Busby KK, Sajatovic M. Review: patient, treatment, and systems-level factors in bipolar disorder nonadherence: a summary of the literature. CNS Neurosci Ther 2010;16:308-315.

47. Cramer JA, Rosenheck R. Enhancing medication compliance for people with serious mental illness. J Nerv Ment Dis 1999; 187:53-55.

48. Velligan DI, Wang M, Diamond P, Glahn DC, Castillo D, Bendle $\mathrm{S}$, et al. Relationships among subjective and objective measures of adherence to oral antipsychotic medications. Psychiatr Serv 2007;58:1187-1192.

49. You JH, Woo YS, Hahn C, Choi JW, Ko HJ. Predicting factors associated with drug compliance in psychiatric outpatients of a university hospital. Korean I Psychopharmacol 2010;21: 29-34.

50. Selvakumar N, Menon V, Kattimani S. A cross-sectional analysis of patterns and predictors of medication adherence in bipolar disorder: single center experience from South India. Clin Psychopharmacol Neurosci 2018;16:168-175.

51. Krueger KP, Felkey BG, Berger BA. Improving adherence and persistence: a review and assessment of interventions and description of steps toward a national adherence initiative. $J \mathrm{Am}$ Pharm Assoc (2003) 2003;43:668-678; quiz 678-679.

52. Fleischhacker WW, Oehl MA, Hummer M. Factors influencing compliance in schizophrenia patients. J Clin Psychiatry 2003;64 Supp/ 16:10-13. 